\title{
Mass estimates for the objects related to class II methanol masers with linear position-velocity structure
}

\author{
Grigorii M. Gos'kov and Andrej M. Sobolev \\ Ural State University, Lenin str. 51, 620083 Ekaterinburg, Russia
}

\begin{abstract}
We study the probability density distributions of mass estimates for class II methanol masers with linear position-velocity structure, of maser cluster, using Monte Carlo tecniques. A uniform random distribution of maser sources along an edge-on rotating circumstellar-ring is assumed as a working hypothesis. It is found that in the cases with sufficiently high number $(>6)$ of observed maser spots it is possible to set upper limits for the mass encircled by the ring. Both turbulent motions and observational uncertainties are shown to result in the broadening of the probability density distribution of the mass estimate. As a consequence, commonly used procedures do not provide correct values for the lower mass estimate. Ten maser objects with the number of observed spots exceeding 6 were chosen for preliminary analysis. Within the framework of the working hypothesis all of these objects were found to be massive $(M>5 M \odot)$ with negligible probability for the mass exceeding $80 M \odot$.
\end{abstract}

\section{Introduction}

In a number of sources the clusters of class II methanol maser spots form a straight line in a position-velocity diagram (Norris et al., 1998, Phillips et al., 1998, Walsh et al. 1998, Minier et al., 1998). This can be interpreted as maser sources distributed in a thin ring within an edge-on circumstellar disk. Such disks are axisymmetric and relatively thin. So, thermodynamic conditions are the most preferable for explaining how the appearance of strong inversion might arise in a rather thin ring. The ring hypothesis was used to derive lower limit for masses of the objects (see citations above). In this contribution we study the influence of intrinsic turbulence and observational uncertainties on the mass estimates and explore the possibility to set upper limits for the probable mass value.

\section{Influence of the number of observed maser spots}

In the case of maser sources distributed along the thin ring the mass encircled by the ring can be derived from the $V_{l s r}$ gradient and the ring radius. Though it is not possible to derive the real value of the ring radius $R$ from observational data, the greatest distance between maser spots $\Delta r$ can be used as a lower limit 
for $R$. So, one can estimate the lower limit of the mass:

$$
M_{e s t}=\left(d V_{l s r} / d r\right)^{2} \cdot(\Delta r)^{3} / G=M \cdot(\Delta r / R)^{3}<M .
$$

For simplicity we assume that the distribution of maser spots along the ring is uniform. In the considered model the accuracy of this estimate depends on the number of spots. We considered large sets of model realizations with 4-12 spots randomly distributed along the ring (2 million realizations for each particular value of the number of spots). It was found that with the number of spots exceeding 4 the value $M_{\text {est }}$ becomes the most probable mass estimate. Figure 1 shows that when the number of spots is sufficiently great the value of $M_{\text {est }}$ becomes quite close to the real value of mass, $M$, in a probabilistic sense. This provides the possibility to set an upper limit for the value of the mass.

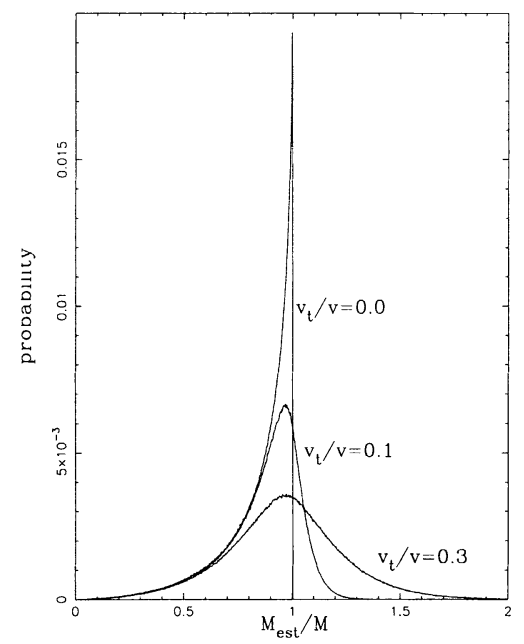

Figure 1. Probability density distributions of the mass estimate considering different number of spots.

\section{Influence of turbulence and uncertainties in spot positions}

Making mass estimates one has to take into account uncertainties in the values of velocity gradient and radius estimates due to turbulence and observational uncertainties.

Indeed, there are considerable turbulent motions in maser formation regions within the disks around young stellar objects. The dispersion of turbulent velocities is usually much greater than the velocity resolution of the observations. In order to study the influence of the turbulence on the mass estimates we calculated a series of model realizations with random deviations in $V_{l s r}$ values. The distribution of deviations was assumed to be normal with the ratio of turbulent velocity dispersion to the linear velocity of the ring rotation, $v_{t} / v$.

In the turbulent case the distribution of maser spots in position-velocity diagram is not strictly linear. In order to reproduce the procedure applied in observational data analysis we used a least squares fit for $d V_{l s r} / d r$ used for 
calculating $M_{\text {est }}$ values. Statistics of our results for the series of 4 million model realizations with 11 spots are presented in the left panel of fig 2 . It is clearly seen that the turbulent motions result in the broadening of the probability density function for mass estimates. It is noteworthy that in the turbulent case some values of $M_{\text {est }}$ exceed the real value of the mass, $M$. Hence, $M_{e s t}$ can not be treated as a lower limit for the mass.

Further, observational uncertainties in the spot positions for many sources are not negligible. Similar to the study of the turbulent case, we calculated a series of models with random deviations in spot positions. Statistics of our results for the series of 4 million model realizations with 11 spots are presented in the right panel of fig 2. It is seen that position uncertainties result in the broadening of the probability density function. Yet, changes in this function are different from the turbulent case. However, we find that this effect has minor importance for the treatment of actual observations.
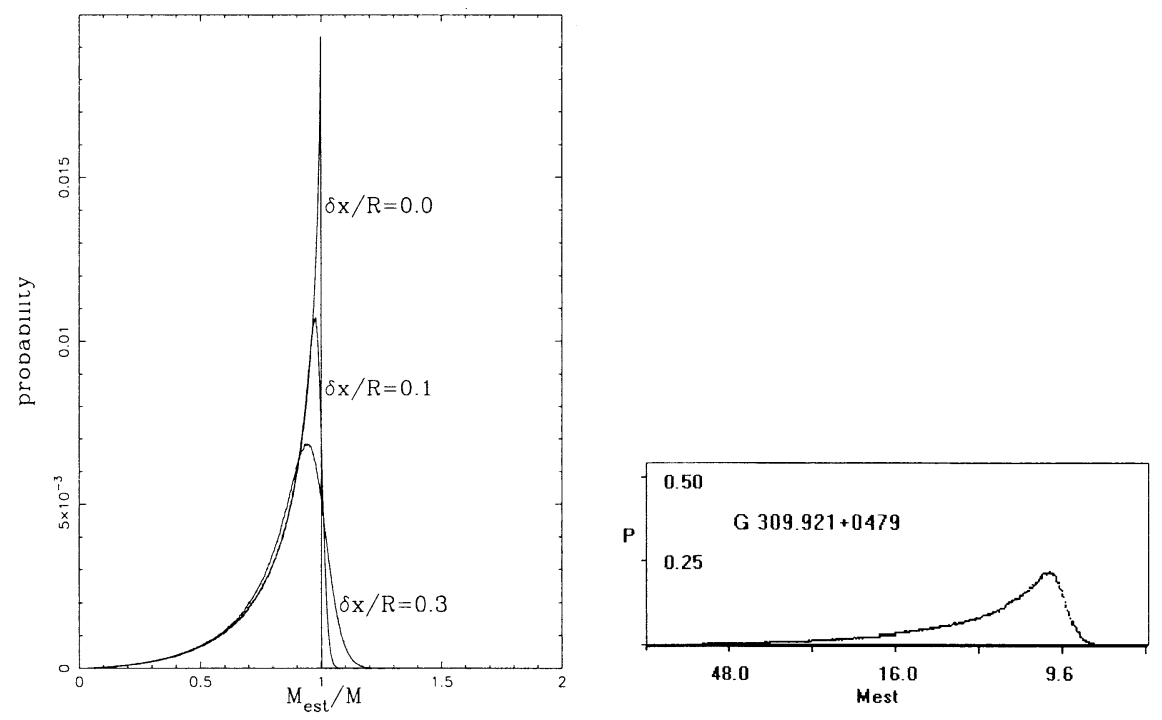

Figure 2. Probability density distributions of the mass estimate for the cases of: different values for turbulent velocity (left panel) and different values of uncertainties in spot positions (right panel).

\section{Application to particular sources}

Available observational information allows construction of the probability density function for particular sources. Indeed, the turbulent velocity dispersion can be estimated from the scatter of observed $V_{l s r}$ values and uncertainties of the maser spot positions which are known. An example for the probability density function can be obtained using the data published in Phillips et al. (1998) and is given in fig 3 .

Probability density functions similar to the one shown in fig 3 . were constructed for 10 sources with linear position-velocity structure: G9.62+0.20, 


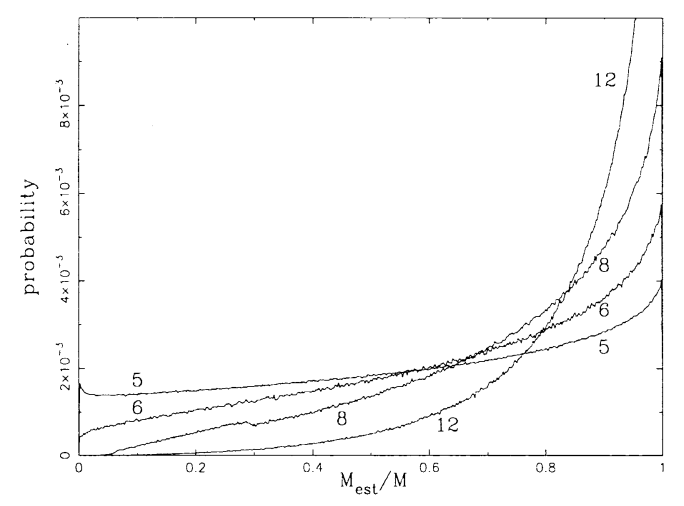

Figure 3. Probability density of the mass estimate for $G 309.92+0.48$ derived from information on 10 maser spots (Phillips et al., 1998).

G305.21+0.21, G309.92+0.48, G316.64-0.09, G318.92-0.17, G323.74-0.26, G331.13-0.24, G337.71-0.05, G339.89-1.26, and G345.01+1.79. For all of these sources the number of spots observed by Phillips et al. (1998) or Walsh et al. (1998) exceeds 6. It was found that the most probable mass estimates for this source sample ranged from 5.6 $M \odot$ for G316.64-0.09 to 75.5 $M \odot$ for G337.710.05 . Only one source had its most probable mass estimate exceeding $30 \mathrm{M} \odot$. Analysis of the combined probability density function shows that the probability of the mass estimates exceeding $80 M \odot$ for our source sample is negligibly small.

\section{Conclusion}

The hypothesis of uniform random distribution of maser sources along edge-on rings is considered. It was found that in the cases with a number of maser spots exceeding 6 it is possible to set upper limit for the mass estimate. Both turbulent motions and observational uncertainties affect the probability density function. Ten maser objects were chosen for preliminary analysis. All of the objects were found to be massive $(M>5 M \odot)$ with a very low probability for the mass exceeding $80 M \odot$.

The current consideration does not account for distance estimate uncertainties and the influence of the inclination of the ring to the line of sight. This analysis is underway.

We thank INTAS (grant 97-11451) and the Russian federal program "Astronomy" for financial support.

\section{References}

Minier V.,Booth R.S.,Conway J.E. 1998, A\&A, 336, L5

Norris R.,Byleveld S.,Diamond P.,Ellingsen S.,etal. 1998, ApJ, 508, 275

Phillips C.,Norris R.,Ellingsen S.,McCulloch P. 1998, MNRAS, 300, 1131

Walsh A.J.,Burton M.G.,Hyland A.R.,Robinson G. 1998, MNRAS, 301, 640 